\title{
AIRP pubblica due libri sul rene policistico
}

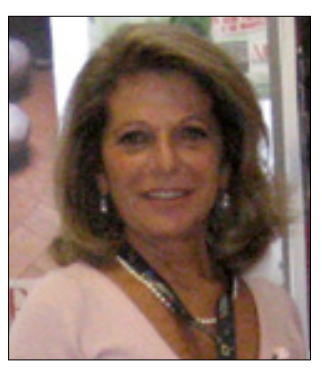

\author{
Luisa Sternfeld Pavia \\ Presidente \\ Associazione Italiana Rene \\ Policistico AIRP onlus \\ luisa.sternfeld.airp@renepolicistico.it
}

Cari lettori, cari amici,

terminato il periodo di meritato riposo estivo, che spero abbiate tutti trascorso nel migliore dei modi, eccoci già in piena attività con la nostra Associazione, con l'energia che ci viene da alcune soddisfazioni che desidero ricordarvi.

Prima di tutto, il successo della nostra campagna di comunicazione "Vogliamo che tutte le passioni possano diventare grandi". Lo scorso 12 Giugno, a Milano, presso l'Auditorium San Fedele, la XVII edizione di Mediastars ha assegnato alla campagna AIRP il Primo Premio nella sezione Radio per la categoria Social-Non Profit. Oltre a questo, ci siamo aggiudicati anche due Special Star, una per il Copy e una per l'Editing.

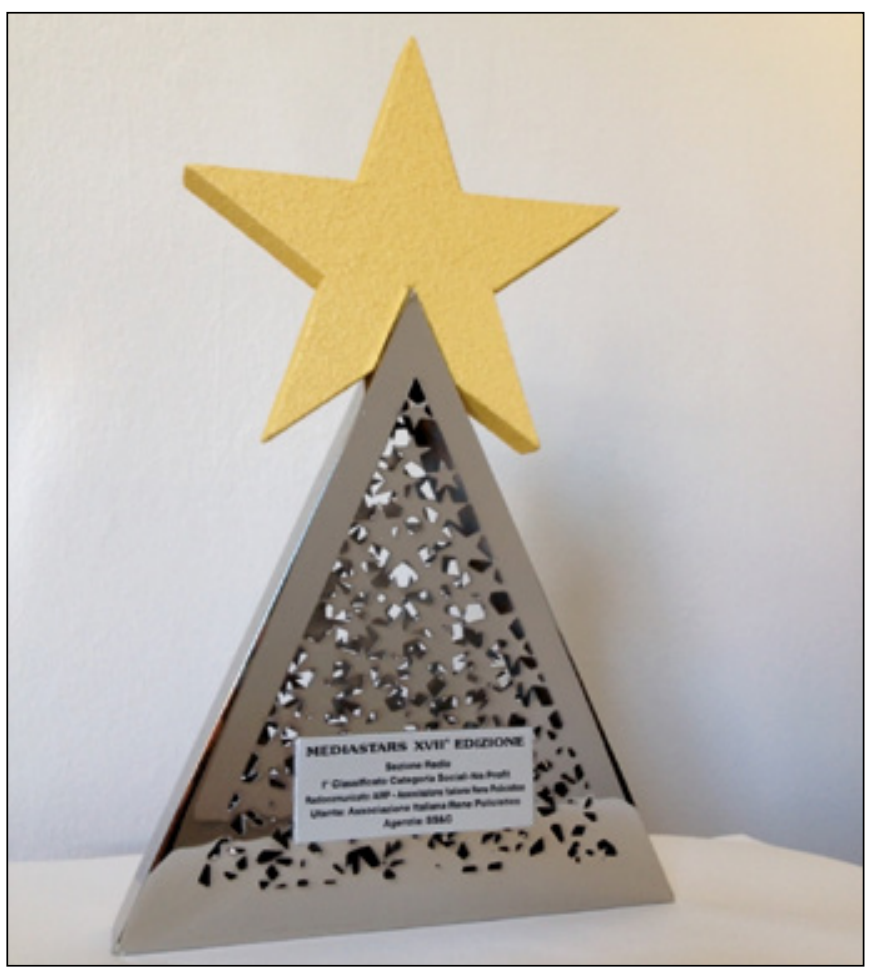

Ringraziamo ancora l'agenzia SS\&C e tutti coloro che hanno collaborato alla realizzazione della campagna. Un riconoscimento che premia il lavoro di tutti e, soprattutto, l'impegno di un'associazione come AIRP, che si adopera per far conoscere la patologia ereditaria del rene policistico.

Ma passiamo alle soddisfazioni del presente. Forse ricorderete che, nello scorso numero della newsletter, avevo accennato a un progetto importante, senza poterlo ancora svelare. Ed ecco di cosa si tratta: AIRP ha curato la pubblicazione di due libri sul rene policistico: uno di carattere divulgativo, per i Pazienti e i loro Familiari e uno di carattere più strettamente scientifico, rivolto ai Medici.

È stato un lavoro lungo e impegnativo, che ci ha visto coinvolti direttamente sia nelle scelte editoriali e grafiche che nelle fasi esecutive finali. Naturalmente, anche in questo caso, ci siamo avvalsi dei professionisti che con passione ci affiancano in tutti i nostri principali progetti.

Il primo si intitola "Rene Policistico Autosomico Dominante. Parlarne è già combatterlo".

Accessibile, facilmente fruibile e dedicato ai pazienti, possiede in sé una grande forza: risponde alla necessità di creare uno strumento che arrivi a tutte le persone affette dalla patologia del rene policistico e all'interno delle famiglie. Infatti, il volume comprende anche un capitolo dedicato ai genitori che vogliono rispondere in modo semplice ma corretto ai quesiti dei bambini sulla malattia. Siamo convinti che raccontare le battaglie quotidiane di tutti i malati di rene policistico, delle loro famiglie e dei medici che li assistono sia il modo migliore per avvicinare il giorno in cui la patologia sarà sconfitta.

Parliamone, quindi, e diffondiamo la conoscenza del rene policistico e del suo impatto individuale e sociale. Discutiamone e portiamo avanti le nostre ragioni in tutti $\mathrm{i}$ luoghi dove può essere utile.

Essere positivi non vuol dire nascondere le difficoltà. Significa affrontarle e decidere che un futuro c'è, se lo vogliamo.

Questo primo volume verrà spedito a tutti i Soci.

Il secondo volume ha come titolo "Manifestazioni renali ed extrarenali del Rene Policistico Autosomico Dominante".

Di impronta più tecnica e specialistica rispetto al precedente, è rivolto a tutta la Comunità Scientifica e nasce dalla necessità di diffondere cultura sulla patologia dell'ADPKD e conoscenze più specifiche su tutte le sue manifestazioni. Il nostro obiettivo è far sì che tutti i malati, affetti dalla patologia del rene policistico, possano trovare nel Medico, sin dai primi sintomi o anche a uno stadio più avanzato della malattia, un vero punto di riferimento, un supporto reale e un'assistenza tempestiva. Siamo certi e fieri di fornire uno strumento concreto di approfondimento, utile anche per chi 


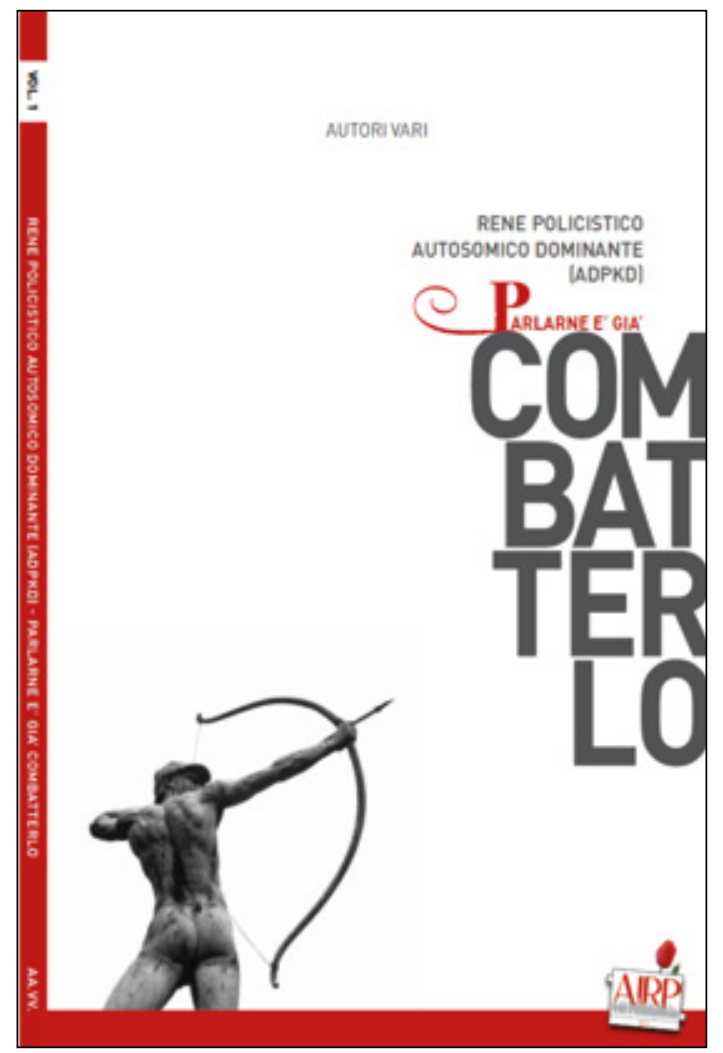

aiuta ogni giorno i pazienti che si trovano a dover combattere la propria battaglia, sperando che, in un futuro non lontano, la malattia possa essere sconfitta.

Un ringraziamento speciale va al Prof. Francesco Scolari che, con questa Lectio Magistralis, arricchisce tutti in modo puntuale e analitico su ogni aspetto della patologia.

I due volumi sono già stampati e il loro debutto è avvenuto al Congresso SIN (Società Italiana di Nefrologia) a Firenze (25/28 Settembre 2013), durante una conferenza stampa dedicata proprio alla loro presentazione e che ha visto la partecipazione degli Autori. Nell'occasione, i volumi sono stati distribuiti ai medici iscritti al Congresso.

Si svolgerà il 5 Ottobre prossimo a Grado, al Palazzo dei Congressi, a conclusione dei lavori del Congresso di Nefrologia "Genetica e Rene" (3-5 Ottobre 2013), la " $2^{a}$ Tavola Rotonda AIRP con i Pazienti" e i loro familiari, per diffondere le conoscenze relative all'ADPKD e all'importanza della prevenzione e per favorire un nuovo tipo di relazione tra Medico e Paziente.

Nel frattempo, prosegue il Road Show "Il Rene Policistico e le sue manifestazioni". La tredicesima tappa è in programma a Torino, per Sabato 12 Ottobre 2013, presso l'unità di Nefrologia, Dialisi e Trapianto dell'Ospedale Molinette. Il Convegno segue lo schema ormai consolidato, che ha incontrato fin qui il gradimento dei partecipanti: una sessione formativa per Medici e Personale Sanitario, con la presenza anche dei Pazienti, e una Tavola Rotonda per favorire il confronto e lo scambio tra Medici, Pazienti e loro Familiari. Anche a Torino verrà illustrato il lavoro di ricerca che AIRP

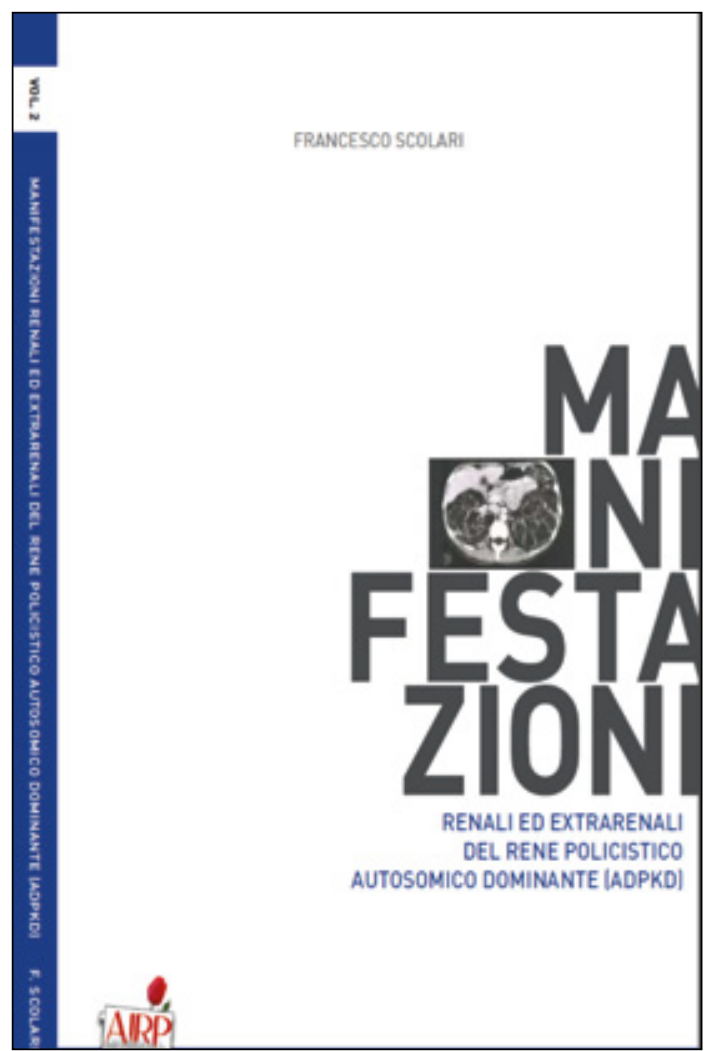

sta finanziando presso l'Ospedale San Raffaele di Milano, dove l'equipe guidata dalla Dr.ssa Alessandra Boletta ha già fatto alcune importanti scoperte. Né mancherà lo Sharing Corner, per registrare i vostri messaggi, che verranno poi postati sulle pagine YouTube e Facebook dell'Associazione. Infine, a proposito dell' Associazione, è importante ricordare che la nostra stessa esistenza dipende dai contributi che riceviamo da voi, anche attraverso le molteplici iniziative che voi stessi organizzate nei vostri territori per finanziare $\mathrm{i}$ progetti e le attività AIRP.

Invito tutti, perciò, a prendere esempio dagli eventi realizzati in particolare a Napoli, a Rimini, ad Ancona, a Milano e in altre località, per ideare e realizzare iniziative analoghe oppure del tutto nuove. Il nostro obiettivo comune, infatti, è di proseguire questo lavoro di sensibilizzazione e di partecipazione intorno alle tematiche della nostra patologia. Per cercare soluzioni e anche per sentirsi meno soli.

Oltre alla consueta rubrica "La voce dei Pazienti", abbiamo pensato di farvi cosa gradita, anticipando su questo numero della nostra rivista il capitolo "La ricerca oggi", tratto dal primo dei due volumi, "Rene Policistico Autosomico Dominante. Parlarne è già combatterlo", redatto dal Dr. Magistroni.

Un caro saluto e buona lettura! 